American Journal of Biochemistry and Biotechnology 2 (2): 80-84, 2006

ISSN 1553-3468

(c) 2005 Science Publications

\title{
Composite Scaffolds for Bone Tissue Engineering
}

\author{
Min Wang \\ Department of Mechanical Engineering, The University of Hong Kong, Pokfulam Road, Hong Kong
}

\begin{abstract}
Biomaterial and scaffold development underpins the advancement of tissue engineering. Traditional scaffolds based on biodegradable polymers such as poly(lactic acid) and poly(lactic acidco-glycolic acid) are weak and non-osteoconductive. For bone tissue engineering, polymer-based composite scaffolds containing bioceramics such as hydroxyapatite can be produced and used. The bioceramics can be either incorporated in the scaffolds as a dispersed secondary phase or form a thin coating on the pore surface of polymer scaffolds. This bioceramic phase renders the scaffolds bioactive and also strengthens the scaffolds. There are a number of methods that can be used to produce bioceramic-polymer composite scaffolds. This paper gives an overview of our efforts in developing composite scaffolds for bone tissue engineering.
\end{abstract}

Key words: bioceramics, composite, scaffolds, bone tissue engineering

\section{INTRODUCTION}

Since the late 1980s, tissue engineering (TE) as a new discipline has made rapid advances ${ }^{[1]}$. Tissue engineering, as a potential medical treatment, holds promises of (a) eliminating re-operations by using biological substitutes, (b) using biological substitutes to solve problems of implant rejection, transmission of diseases associated with xenografts and allografts, and shortage in organ donation, (c) providing long-term solutions in tissue repair or treatment of diseases, and (d) potentially offering treatments for medical conditions that are currently untreatable. Tissue engineering has therefore attracted great attention in science, engineering, medicine, and the society ${ }^{[2]}$.

It is now generally recognised that one of the key issues in tissue engineering is the development of suitable biodegradable materials and scaffolds for seeding cells and for the subsequent growth of tissues ${ }^{[3]}$. For bone tissue engineering, major issues of tissue engineering scaffolds include the use of appropriate matrix materials for scaffolds, control of porosity and pore characteristics of scaffolds, mechanical strength of scaffolds, scaffold degradation properties, and bioactivity (i.e., osteoconductivity, or osteoinductivity if possible) of scaffolds. Biomaterials experience with biodegradable polymers in the pre-TE time (before $1987^{[4]}$ ) has heavily guided researchers towards using medical-profession-accepted and the US Food and Drug Administration (FDA)-approved biopolymers such as poly(lactic acid) (PLA) and poly(lactic acid-co-glycolic acid) (PLGA) for constructing tissue engineering scaffolds. In recent years, other polymers have also been developed for tissue engineering applications ${ }^{[5]}$. Despite their phenomenal developments in the past three decades ${ }^{[6]}$, bioactive bioceramics (including glasses and glass-ceramics) have been overlooked for tissue engineering applications until recently. It has been shown that some bioactive glasses have the potential for bone tissue engineering ${ }^{[7]}$ and there are now reports on the development of tissue engineering scaffolds purely made of bioceramics ${ }^{[8,9]}$.

Compared to the strengths of metals and ceramics for medical applications, the strengths of biodegradable polymers are already very low. With the introduction of pores in the polymers to form tissue engineering scaffolds, the strengths of porous structures are further decreased, as strengths of materials decrease drastically with an increase in porosity ${ }^{[10]}$. On the other hand, polymers such as PLA and poly( $\varepsilon$-caprolactone) (PCL) are considered to be non-osteoconductive. In order to seek better scaffolds for bone tissue engineering, a composite strategy can therefore be adopted ${ }^{[11]}$. Polymer-based scaffolds containing bioactive bioceramics can be produced in which the bioceramics can serve two purposes: (a) making the scaffolds osteoconductive, and (b) reinforcing the scaffolds. With this composite strategy, there are two approaches for making bioceramic-polymer composite scaffolds:

(1) incorporating bioceramic particles in the scaffold through a variety of techniques; and

(2) coating a polymer scaffold with a thin layer of apatite through biomimetic processes.

Both strategies have been employed in our efforts to develop usable scaffolds for bone tissue engineering.

\section{COMPOSITE SCAFFOLDS}

\section{Particulate Bioceramics for Composite Scaffolds}

Particulate materials of hydroxyapatite (HA), tricalcium phosphate (TCP) and a few bioactive glasses

Corresponding Author: Dr. M. Wang, Department of Mechanical Engineering, The University of Hong Kong, Pokfulam Road, Hong Kong Tel: +852 2859 7903, Fax: +852 28585415 
can be used in composite scaffolds for achieving osteoconductivity ${ }^{[11]}$. In one of our early investigations, plasma-sprayed HA (psHA) particles were produced and used, as particulate psHA was found to be more bioactive than highly crystalline HA particles due to the amorphous component in psHA particles ${ }^{[12]}$. These spherical psHA particles were in the micrometer size range (Fig.1a). In our recent investigations, carbonated HA (CHA) particles were synthesized through a nanoemulsion process ${ }^{[13]}$. CHA is also more soluble and bioactive than highly crystalline HA due to the $\mathrm{CO}_{3}{ }^{2-}$ substitution in HA lattice ${ }^{[6]}$. Furthermore, the spherical CHA particles were in the nanometer size range (Fig.1b) and are thus more suitable for incorporation into polymers to form composite scaffolds.

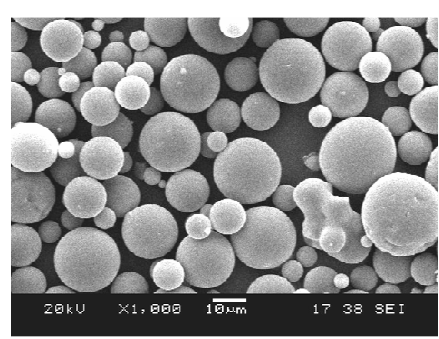

(a) psHA microspheres

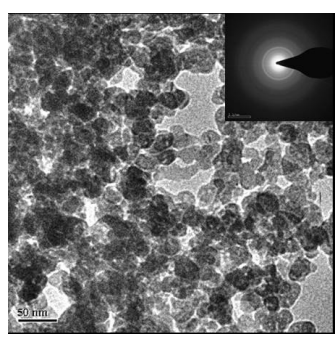

(b) CHA nanospheres
Fig. 1: Particulate HA for composite scaffolds

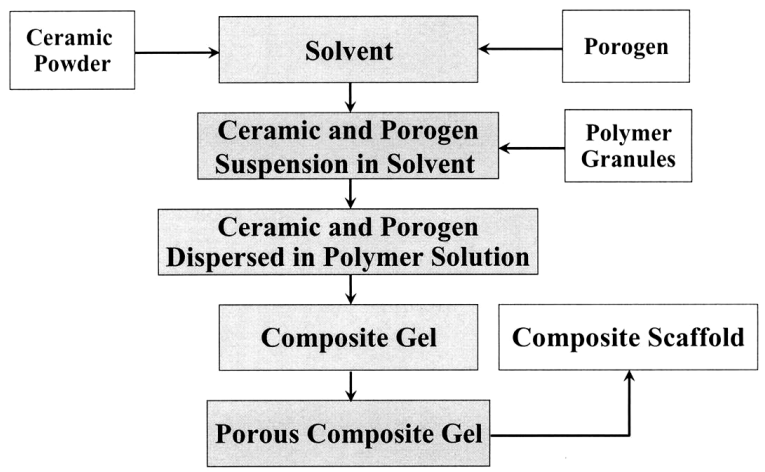

Fig. 2: Manufacture of bioactive composite scaffolds

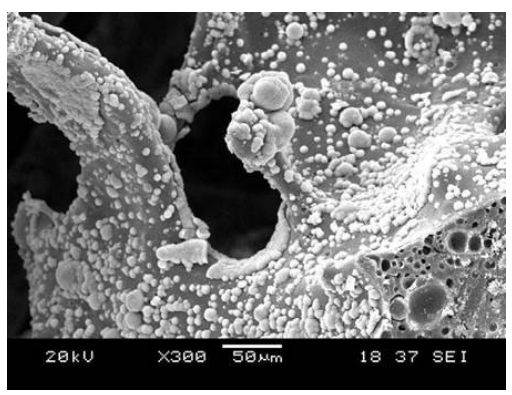

Fig. 3: Formation of bone-like apatite on a psHA/PLLA composite scaffold through immersion in a simulated body fluid

Incorporating Bioceramic Particles in the Scaffolds

In our early investigations, psHA particles were incorporated in a poly(L-lactic acid) (PLLA) to form osteoconductive composite scaffolds ${ }^{[14]}$. The manufacturing process of these scaffolds is shown in Fig.2. In this process, freeze-drying was used at the final stage to produce the porous structure. Just as in the production of polymer scaffolds ${ }^{[15]}$, for composite scaffolds, factors such as polymer solution concentration, porogen type and size, freeze-drying parameters, etc. play very important roles in forming the scaffolds of desired porous structures (pore geometry, pore size and size distribution, pore interconnectivity, thickness of pore walls, etc.) and hence the mechanical performance. It was shown through in vitro experiments that the composite scaffolds possessed bioactivity due to the incorporation of psHA particles (Fig.3).

In a recent study, it was shown that using an emulsion freeze-drying process, composite scaffolds based on polyhydroxybutyrate (PHB) and polyhydroxybutyrate-co-valerate (PHBV) natural polymers could be produced ${ }^{[16]}$. In these scaffolds, the CHA nanospheres (Fig.1b) were incorporated. Through this process, highly porous structure with a controlled pore morphologies could be achieved (Fig.4).

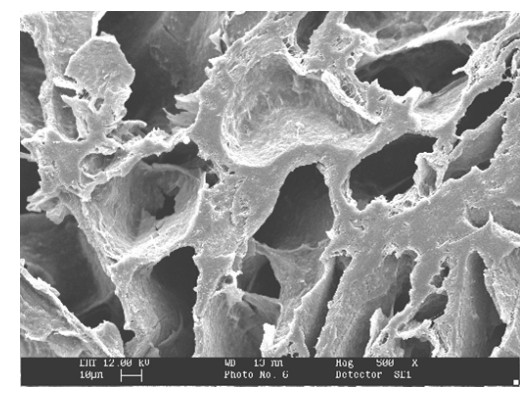

Fig. 4: CHA/PHBV composite scaffold produced through an emulsion freeze-drying process

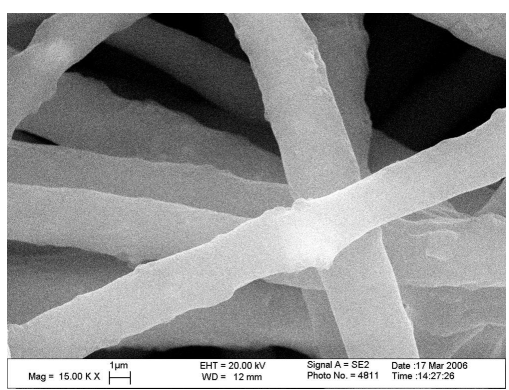

Fig. 5: CHA/PHBV composite nanofibers produced through electrospinning

In recent years, biodegradable fibers which have diameters in the nanometer range have attracted great attention, as it is considered that the proper in vivo phenotype cannot be consistently achieved if cells are presented with fibers with diameters equal to or greater than the cell size. Among technologies for making polymer fibers of nanometer dimensions, electrospinning is the most appealing and the process is relatively simple. A study was recently conducted on 
electrospinning of PHB and PHBV polymers and their composites ${ }^{[17]}$. It was found that electrospinning parameters (polymer solution concentration, solution feeding rate, injection needle diameter, electrical voltage, and working distance) all had significant effects on the resultant fibers. With careful experimentation, $\mathrm{CHA} / \mathrm{PHBV}$ composite nanofibers containing CHA nanospheres could be produced (Fig.5). The incorporation of CHA nanospheres was confirmed through EDX analyses of the fibers.

Rapid prototyping (RP) technologies have become increasingly popular for producing tissue engineering scaffolds, as there are a few distinctive advantages of using RP technologies. One of our efforts is to use the selective laser sintering (SLS) technology to manufacture CHA/PLA composite scaffolds ${ }^{[18]}$. In the SLS route, there are two major issues: (a) obtaining powders of appropriate characteristics for sintering, and (b) minimizing the use of sintering powder. The latter issue could be solved by building and installing a miniature sintering platform in a commercial SLS machine. The former issue required the production of composite powders of suitable characteristics. Through a microemulsion process, both PLA and CHA/PLA composite powders were made (Fig.6).

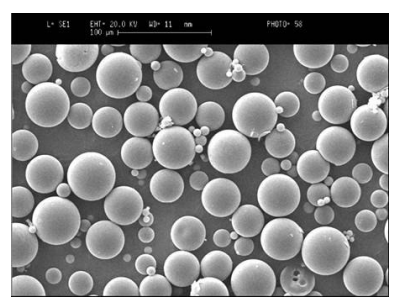

(a) PLA microspheres

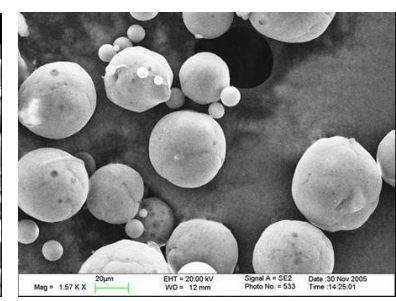

(b) CHA/PLA microspheres
Fig. 6: Powders for producing composite scaffolds through selective laser sintering

\section{Coating the Polymer Scaffold with an Apatite Layer}

As stated earlier, scaffolds made purely of biodegradable polymers such as PLA and PLGA are not osteoconductive. To impart bioactivity on the polymer scaffolds and also strengthen the scaffolds, apatite coatings could be formed on pores surfaces of the scaffolds before they are put in use for tissue engineering. For polymers, forming apatite coatings on their surfaces can be achieved through biomimetic processes. However, as polymers such as PLA degrades fast in the aqueous environment, normal biomimetic processes that have been developed for coating apatite on metals or ceramics ${ }^{[19]}$ are not suitable for these polymers. Therefore, an accelerated biomimetic process was developed to coat PLLA scaffolds with a thin layer of apatite ${ }^{[20,21]}$. Apatite was found to form on PGA meshes and PLLA scaffolds (Fig.7), and with a dynamic environment, biomimetic apatite coatings could be produced on pores inside the scaffolds. Further investigations revealed that a composite coating consisting of nano-sized apatite crystals and collagen which mimics bone structure could also be produced on PLLA scaffolds (Fig.8). It was found that the apatite coating and apatite/collagen composite coating could improve interactions between osteoblasts and PLLA scaffolds and that the composite coating was more effective than apatite coating in improving such interactions ${ }^{[22]}$. Fig.9 shows that osteoblast-like cells could attach and proliferate on the apatite/collagen coating formed on PLLA via the biomimetic process.

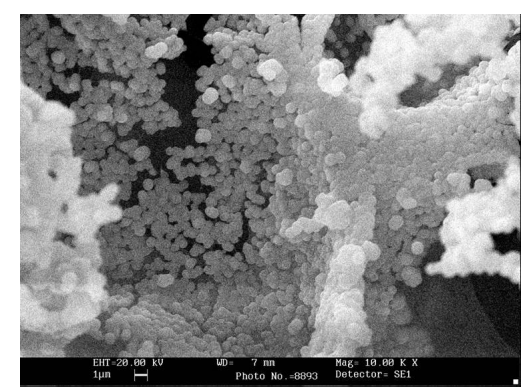

Fig. 7: Bone-like apatite coating formed on the pore surface of a PLLA scaffold through an accelerate biomimetic process

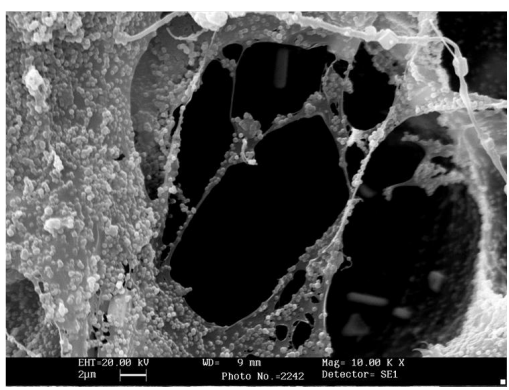

Fig. 8: Apatite/collagen composite coating formed on the pore surface of a PLLA scaffold through an accelerate biomimetic process

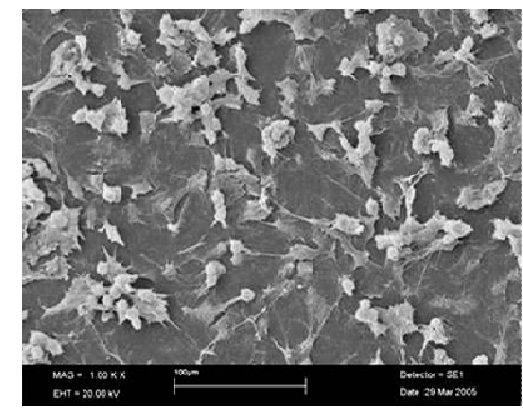

Fig. 9: Osteoblast-like cells attaching to the apatite/collagen composite coating

\section{CONCLUDING REMARKS}

Currently, there are a number of strategies for developing bone tissue engineering scaffolds. The composite strategy provides a means for achieving stronger and bioactive scaffolds as compared to conventional polymer scaffolds. The two approaches in 
the composite route result in scaffolds of different, distinctive characteristics. Bioceramics obviously have an important role to play in constructing osteoconductive tissue engineering scaffolds. Systematic studies are required for the manufacture, characterisation, and in vitro and in vivo assessments of bioceramic-polymer composite scaffolds. Quite often, the scaffold manufacturing technology is selected together with considerations of choosing appropriate constituting materials of the composite. Osteoconductive composite scaffolds appear to have great potential for bone tissue engineering.

\section{ACKNOWLEDGEMENTS}

The author thanks his research staff and students in Nanyang Technological University, the Hong Kong Polytechnic University and the University of Hong Kong for conducting the experimental work. He also thanks his collaborators for their support. He is grateful to funding agencies/universities in Singapore and Hong Kong for funding his research on tissue engineering.

\section{REFERENCES}

1. Vacanti JP, Vacanti CA, (2000). The history and scope of tissue engineering. in: Lanza RP, Langer R, Vacanti J, (eds), Principles of Tissue Engineering, Academic Press, San Diego, 3-7

2. Lysaght MJ, Hazlehurst AL, (2004). Tissue engineering: The end of the beginning. Tissue Engineering, 10: 309-320

3. McIntire LV, (ed), (2003). WTEC Panel on Tissue Engineering Research: Final Report, Academic Press, San Diego

4. Skalak R, Fox CF, (eds), (1988). Tissue Engineering, Alan R.Liss Inc., New York

5 Kohn J, Abramson S, Langer R, (2004). Bioresorbable and bioerodible materials. in: Ratner BD, Hoffman AS, Schoen FJ, Lemons JE, (eds), Biomaterials Science: An Introduction to Materials in Medicine, Academic Press, San Diego, 115-127

6. Wang M, (2002). Bioceramics. in: Ikada Y, (ed), Recent Research Developments in Biomaterials, Research Signpost, Trivandrum, 33-76

7. Huygh A, Schepers EJG, Barbier L, Ducheyne P, (2002). Microchemical transformation of bioactive glass particles of narrow size range, a 0-24 months study. J Mater Sci: Mater Med, 13: 315-320

8. Callcut S, Knowles JC, (2002). Correlation between structure and compressive strength in a reticulated glass-reinforced hydroxyapatite foam. $J$ Mater Sci: Mater Med, 13: 485-489

9. Pereira MM, Jones JR, Hench LL, (2005). Bioactive glass and hybrid scaffolds prepared by sol-gel method for bone tissue engineering. Advances in Applied Ceramics, 104: 35-42
10. Gibson LJ, Ashby MF, (1997). Cellular Solids: Structure and Properties, Cambridge University Press, Cambridge

11. Wang M, (2003). Developing bioactive composite materials for tissue replacement. Biomaterials, 24: 2133-2151

12. Weng J, Wang M, Chen J, (2002). Plasma sprayed calcium phosphate particles with high bioactivity and their use in bioactive scaffolds. Biomaterials, 23: 2623-2629

13. Zhou WY, Wang M, Cheung WL, Gao BC, Jia DM, (2006). Synthesis of carbonated hydroxyapatite nanospheres through nanoemulsion. submitted for publication

14. Wang M, Chen LJ, Ni J, Weng J, Yue CY, (2001). Manufacture and evaluation of bioactive and biodegradable materials and scaffolds for tissue engineering. J Mater Sci: Mater Med, 12: 855-860

15. Weng J, Wang M, (2001). Producing chitin scaffolds with controlled pore size and interconnectivity for tissue engineering. J Mater Sci Lett, 20: 1401-1403

16. Sultana N, Wang M, (2006). Fabrication and characterisation of polymer and composite scaffolds based on polyhydroxybutyrate and polyhydroxybutyrate-co-hydroxyvalerate. Key Engineering Materials, accepted

17. Tong HW, Wang M, (2006). Effects of processing parameters on the morphology and size of electrospun PHBV micro- and nano-fibers. Key Engineering Materials, accepted

18. Zhou WY, Lee SH, Cheung WL, Wang M, Ip WY, (2006). Selective laser sintering of porous scaffolds from poly(L-lactide) microspheres and its nanocomposite with carbonated hydroxyapatite nanospheres. ESB2006, Nantes, France, accepted

19. Kokubo T, Kim HM, Kawashita M, Nakamura T, (2004). Bioactive metals: preparation and properties. J Mater Sci: Mater Med, 15: 99-107

20. Chen Y, Mak AFT, Wang M, (2005). Accelerated formation of bone-like apatite on biodegradable polymer substrates. Key Engineering Materials, 284: 509-512

21. Chen Y, Mak AFT, Li J, Wang M, Shum AWT, (2005). Formation of apatite on poly( $\alpha$-hydroxy acid) in an accelerated biomimetic process. $J$ Biomed Mater Res Pt B, 73B: 68-76

22. Chen Y, Mak AFT, Wang M, Li J, Wong MS, (2006). PLLA scaffolds with biomimetic apatite coating and biomimetic apatite/collagen composite coating to enhance osteoblast-like cells attachment and activity. Surface \& Coatings Technology, in press 